\title{
Conhecimento do consenso de sepse por médicos residentes de um hospital universitário
}

\author{
Knowledge of the sepsis consensus by resident physicians of a university hospital \\ Rilva Lopes de Sousa Muñoz ${ }^{* *}$; Graziela Batista de Sousa*; Carolina Campos de Brito*; Bruno \\ D’Paula ${ }^{\S}$; Filipe Siqueira Teixeira ${ }^{\S}$;Rayanne Pereira Cabral ${ }^{\S}$;
}

Como citar esse artigo. Muñoz, RLS De Sousa, GB; De Brito, CC; D’Paula, B Teixeira,FS;Cabral,RP.Conhecimentodo consenso de sepse por médicos residentes de um hospital universitário. Revista de Saúde. 2018 Jul./Dez.; 09 (2): 09-15.

\begin{abstract}
Resumo
O conhecimento dos critérios de consenso de sepse pelos médicos tem grande relevância por propiciar maior oportunidade de diagnóstico de um quadro grave e de alta mortalidade. O objetivo deste estudo foi avaliar o conhecimento do consenso de sepse por médicos residentes de um hospital universitário. Foi avaliado o conhecimento de 59 médicos do Programa de Residência Médica do Hospital Universitário Lauro Wanderley (HULW), por meio da aplicação de um questionário de avaliação com base no consenso de sepse. A média de idade dos residentes foi de 28 anos, sendo $61,2 \%$ do sexo feminino. A maioria dos residentes do HULW refere ter conhecimento acerca do Consenso de Sepse, que colocam em prática em suas atividades cotidianas, em um processo de aperfeiçoamento profissional, que constitui o objetivo principal da residência médica. Contudo, a avaliação do conhecimento sobre sepse revelou que os percentuais de respostas corretas em parâmetros clínicos e laboratoriais foram insatisfatórios, sobretudo em relação aos parâmetros clínicos e ao conceito de choque séptico. Conclui-se que, embora quase todos os residentes entrevistados $(91 \%)$ tenham referido conhecer o consenso sobre sepse, os percentuais de acertos foram insatisfatórios, sobretudo em relação ao choque séptico.
\end{abstract}

Palavras-chave: Síndrome de Resposta Inflamatória Sistêmica; Sepse; Residência Médica.

\begin{abstract}
The knowledge of the consensus criteria of sepsis by physicians has great relevance for providing greater opportunity for diagnosis of a severe and high mortality. The objective of this study was to evaluate the knowledge of the consensus of sepsis by physicians living in a university hospital. A total of 59 physicians from the Residency Program of the Lauro Wanderley University Hospital were evaluated through an evaluation questionnaire based on the consensus of sepsis. The mean age of the residents was 28 years, $61,2 \%$ female. The majority of HULW residents report having knowledge about the Sepsis Consensus, acquired mainly in undergraduate studies, and that they put it into practice in their daily activities, in a process of professional improvement that is the main objective of medical residency. However, the evaluation of the knowledge about sepsis reveals that the percentages of correct answers in clinical and laboratory parameters were unsatisfactory, o especially in relation to clinical parameters and to the concept of septic shock. It was concluded that, although almost all residents interviewed (91\%) reported having known about the consensus on sepsis, the percentage of correct answers was unsatisfactory, especially in relation to septic shock.
\end{abstract}

Keywords: Systemic Inflammatory Response Syndrome; Sepsis; Medical Residency.

\section{Introdução}

A sepse é definida como um conjunto de anormalidades fisiológicas, patológicas e bioquímicas, cuja expressão primordial é a disfunção de órgãos com risco de vida, a partir de uma resposta desregulada do hospedeiro a uma infecção. Esta condição representa um desafio para médicos de praticamente todas as especialidades, dada a necessidade de pronto reconhecimento e tratamento oportuno ${ }^{2,3}$.

Tem havido uma crescente preocupação das instituições hospitalares com a adequada utilização de diretrizes clínicas e instrumentos gerenciais capazes de elevar o nível de qualidade e resolutividade de seus serviços $^{4}$. Nesse sentido, o domínio do conhecimento dos critérios de definição de sepse pelos médicos tem grande relevância por propiciar maior oportunidade de diagnóstico ${ }^{1}$. Mesmo os profissionais não diretamente envolvidos em atendimento rotineiro de pacientes críticos devem ser capazes de reconhecer os sintomas e sinais de sepse e providenciar o encaminhamento imediato para que o tratamento possa ser feito. Assim, se justifica a busca de evidências empíricas sobre a amplitude desse conhecimento por médicos residentes

\footnotetext{
Afiliação dos autores: Centro de Ciências Médicas, Universidade Federal da Paraíba, João Pessoa, Paraíba, Brasil

† Docente do Departamento de Medicina Interna do Centro de Ciências Médicas da Universidade Federal da Paraíba (UFPB)

${ }^{\star}$ Médicas residentes do Hospital Universitário Lauro Wanderley (UFPB)

§ Alunos de graduação em medicina da UFPB

*rilva@ccm.ufpb.br
} 
e sua aplicação no meio profissional, sobretudo quando se leva em conta que a assistência no que concerne ao combate à sepse nesta região ainda não é efetiva ${ }^{4,5}$.

Em 1991, na Conferência Internacional de Consenso de Sepse (American College of Chest Physicians Society - ACCP e Society of Critical Care Medicine - SCCM) definiu-se a Síndrome da Resposta Inflamatória Sistêmica (SIRS). O entendimento do Instituto Latino-Americano de Sepse (ILAS) estava fundamentado nestes parâmetros do Consenso da ACCP/ SCCM, que se supunha permitir o estabelecimento de uma definição universal das manifestações da resposta inflamatória à infecção, facilitando seu reconhecimento e tratamento precoces ${ }^{6}$. A definição de sepse foi atualizada em 2016 após uma nova conferência de consenso, publicando-se os últimos critérios definidores de sepse ${ }^{1}$, conhecidos como "Sepsis 3". Embora as definições tenham sido endossadas por muitas sociedades de terapia intensiva em todo o mundo, também geraram controvérsias, principalmente no que se refere ao aumento da especificidade à custa de redução da sensibilidade dos novos critérios? ${ }^{7}$.

Supõe-se que muitos médicos ainda desconhecem as definições anteriores às dos critérios de Sepsis 3, considerando que um quarto dos pacientes com sepse ainda recebe tratamento inapropriado e apresenta piora de seuprognóstico em consequência do atrasodiagnóstico ${ }^{8,9}$. Assim, no presente estudo, de acordo com a orientação do Instituto Latino-americano de Sepse ${ }^{6,10}$, mantiveramse os critérios operacionais utilizados anteriormente, os quais eram vigentes à época da execução deste estudo, e que são considerados mais adequados para aplicação em países em desenvolvimento ${ }^{11-14}$. É relevante verificar a magnitude em que estes critérios de diagnóstico de sepse eram conhecidos, aplicados e compatíveis com o consenso então vigente para identificar a sua aceitação entre residentes em treinamento em serviço.

Assim, o presente estudo teve como objetivo avaliar o conhecimento dos médicos residentes de um hospital universitário sobre o consenso internacional de sepse vigente até 2016 .

\section{Material e métodos}

Este estudo teve modelo observacional e transversal. A amostragem foi de natureza nãoprobabilística e intencional. A população-alvo incluiu todos os médicos residentes que desenvolveram suas atividades de treinamento em serviço no Hospital Universitário Lauro Wanderley (HULW), na cidade de João Pessoa, Estado da Paraíba, no período entre agosto de 2015 e julho de 2016.

Por ter sido realizado imediatamente antes da publicação do Consenso Sepsis 3, a definição operacional das variáveis na presente pesquisa ocorreu da seguinte forma, segundo o Instituto Latino Americano de Sepse1:

- SIRS: resposta do organismo a uma lesão variada (trauma, pancreatite, grande queimado, infecção sistêmica), com a presença de pelo menos dois dos seguintes critérios: febre (temperatura corporal acima de $38{ }^{\circ} \mathrm{C}$ ) ou hipotermia (temperatura corporal menor que $36{ }^{\circ} \mathrm{C}$ ); taquicardia (frequência cardíaca acima de $90 \mathrm{bpm}$ ); taquipneia (frequência respiratória acima de $20 \mathrm{rpm}$ ) ou $\mathrm{PaCO} 2$ menor que $32 \mathrm{mmHg}$; leucocitose (leucócitos acima de 12.000 células $/ \mathrm{mm}^{3}$ ) ou leucopenia (leucócitos abaixo de 4.000 células $/ \mathrm{mm}^{3}$ ), ou leucograma com mais de $10 \%$ de bastões.

- Sepse: SIRS decorrente de um processo infeccioso confirmado ou suspeito.

- Sepse grave: infecção suspeita ou confirmada associada a disfunção orgânica, de forma independente da presença de sinais de SRIS.

- Choque séptico: Sepse que evoluiu com hipotensão não corrigida com reposição volêmica (PAM $\leq 65 \mathrm{mmHg}$ ), de forma independente de alterações de lactato.

Nos procedimentos de coleta de dados, inicialmente foi solicitada à Comissão de Residência Médica do HULW a listagem de todos os médicos residentes que atuavam no hospital em agosto de 2015, no primeiro, segundo e terceiros anos das residências médicas, totalizando 90 médicos. Após obtenção da listagem contendo os nomes de todos os médicos residentes do HULW, foi feito o convite para que participassem da pesquisa nas próprias enfermarias do hospital, após esclarecimento sobre o estudo e assinatura do Termo de Consentimento Livre e Esclarecido. Um questionário impresso e sem identificação do participante foi entregue aos médicos residentes para que o respondessem prontamente.

$\mathrm{O}$ instrumento de pesquisa foi um questionário adaptado de estudo anterior ${ }^{11}$, fundamentado nas definições estabelecidas no Consenso da ACCP/SCCM, referendado no Consenso Brasileiro de Sepse e no entendimento da ILAS ${ }^{6,13}$. Trata-se de um questionário semiestruturado e auto-administrado, contendo informações demográficas e questões sobre as definições de SIRS, sepse, sepse grave e choque séptico.

Nas questões numeradas como 2 e 3 do questionário, o entrevistado foi orientado a assinalar respectivamente os três parâmetros clínicos e um laboratorial que definiam a SIRS e completá-los com os seus respectivos valores corretos. Como estas duas questões são relacionadas ao conhecimento dos critérios de definição de SIRS, os acertos foram analisados de forma conjunta. Neste item referente à SIRS, solicitou-se o preenchimento dos valores referentes aos parâmetros clínicos (frequência cardíaca, frequência respiratória, pressão arterial, temperatura e diurese) e laboratoriais (valores gasométricos, hematimétricos, lactato e 
creatinina). Foi considerado acerto parcial da questão quando os valores foram assinalados incorretamente, juntamente com valores corretos. Os mesmos critérios foram aplicados à análise das questões 5,6 e 7, relacionadas ao conhecimento dos critérios de definição de sepse ${ }^{12}$.

Quanto ao resultado do preenchimento dos itens sepse grave e choque séptico, consideraramse apenas dois desfechos (resposta correta/resposta incorreta), em virtude da existência de apenas uma alternativa a ser assinalada nestas duas classificações. No item referente à sepse grave, as alternativas eram hipotensão; hipotensão e hipoperfusão; hipotensão e/ou hipoperfusão; hipoperfusão, enquanto no item do choque séptico, só uma alternativa deveria ser assinalada entre as seguintes: hipotensão refratária a volume; hipotensão refratária a volume e hipoperfusão; hipotensão refratária a volume e/ou hipoperfusão; hipotensão grave.

As adaptações realizadas no questionário original foram as seguintes: na caracterização do participante, a adição da especialidade da residência, duração da residência e questões relativas ao conhecimento sobre SIRS (existência desse conhecimento, momento em que o obteve, aplicação na prática clínica durante a residência no HULW).

O estudo foi aprovado pelo Comitê de Ética em Pesquisa do HULW sob parecer no 1.152.682, de 30/06/2015.

\section{Resultados}

Abordaram-se 69 médicos residentes da listagem de 90 potenciais participantes. Entre os 69 residentes convidados a participar, 67 aceitaram compor a amostra da pesquisa e receberam o questionário para preenchimento. Dos questionários aplicados, oito estavam preenchidos de forma incompleta e foram excluídos, resultando no tamanho amostral final de 59 questionários, o que representou $65,5 \%$ da população de médicos residentes do HULW no período da realização da pesquisa.

Amédia de idade dos 59 residentes foi de 28 anos $( \pm$ 2,3 anos), $61,2 \%$ do sexo feminino, $49,3 \%$ encontravamse no primeiro ano da residência, $32,8 \%$ no segundo e $17,9 \%$ no terceiro. O maior número de participantes da pesquisa foi da residência em clínica médica $(28,4 \%$ da amostra), seguida pelos da cirurgia geral $(13,4 \%)$, pediatria $(11,9 \%)$ e ginecologia e obstetrícia $(11,9 \%)$. Os residentes de outras especialidades representaram $31,5 \%$ da composição da amostra: oftalmologia (9\%); dermatologia (7,5\%); anestesiologia (6\%); infectologia $(4,5 \%)$; endocrinologia (3\%); endoscopia ginecológica e reumatologia somadas representaram $1,5 \%$ da amostra. $\mathrm{O}$ número de participantes de cada especialidade na amostra apresentou proporcionalidade em relação ao quantitativo de residentes, considerando-se os maiores percentuais nas áreas básicas.

Em relação à pergunta sobre o conhecimento do Consenso de Sepse, 91\% dos residentes responderam afirmativamente, sendo que $52(88,1 \%)$ informaram que este conhecimento foi obtido durante seu curso de graduação, enquanto $7(11,9 \%)$ passaram a conhece-lo após o ingresso na residência médica. No que se refere à aplicação deste Consenso de Sepse então vigente, 52 $(88,1 \%)$ referiram que o empregavam na sua prática de treinamento durante a residência médica.

Mais de cinco casos classificados como SIRS foram acompanhados na prática pela maior parte dos médicos $(58,1 \%)$. A maioria dos casos de sepse diagnosticada foi vista tanto nas enfermarias quanto na unidade de terapia intensiva do HULW $(73,1 \%)$, enquanto $26,9 \%$ responderam ter diagnosticado os casos apenas no setor das enfermarias. Entre os residentes que referiram ter diagnosticado e acompanhado casos definidos conforme o consenso, $11,9 \%$ afirmaram terem sido casos classificados como SIRS, enquanto $55,2 \%$ referiram os casos diagnosticados como sepse, $40,3 \%$ como sepse grave e $23,9 \%$ como choque séptico.

Os resultados do preenchimento do questionário de avaliação do conhecimento sobre SIRS/sepse revelaram percentuais de respostas incorretas em parâmetros clínicos e laboratoriais que variaram de $24,5 \%$ a $39 \%$, respectivamente, observando-se mais respostas corretas em relação aos parâmetros laboratoriais que clínicos (Tabela 1).

A maior parte das respostas referentes à definição de sepse grave e choque séptico foi incorreta (Tabela 2).

As pontuações obtidas pelos médicos residentes através das respostas ao questionário de avaliação do conhecimento do Consenso de Sepse por cada especialidade revelaram mais acertos entre os residentes da clínica médica ( $83 \%$ dos 18 residentes) e pediatria (87,5\% dos 8 residentes) (Tabela 3 ).

Ao se compararem os anos de residência com os acertos nos itens do questionário referentes ao conceito de sepse grave e choque séptico, verificou-se maior êxito entre os residentes do segundo ano, entretanto com significância estatística apenas em relação ao conceito de sepse grave $(\mathrm{p}=0,03)$ através do teste de KruskalWallis.

\section{Discussão}

Quase todos os residentes entrevistados referiram conhecer o consenso de sepse vigente à época da coleta de dados deste estudo (segundo semestre de 2015), e que, antes de 2016, coincidiam com os critérios aceitos pelo Instituto Latino-Americano de Sepse $^{6}$. Esta instituição considera atualmente que "não será viável o 
Tabela 1. Resultados do preenchimento de critérios clínicos e laboratoriais de SIRS e sepse de acordo com o Consenso de Sepse pelos médicos residentes $(\mathrm{n}=59)$

\begin{tabular}{lccc}
\hline Critérios de SIRS e sepse & $\begin{array}{c}\text { Resultados } \\
\text { Correto }\end{array}$ & $\begin{array}{c}\text { Parcialmente correto } \\
\mathbf{f}(\%)\end{array}$ & $\begin{array}{c}\text { Incorreto } \\
\mathbf{f}(\%)\end{array}$ \\
\hline $\begin{array}{l}\text { SIRS } \\
\text { Parâmetros clínicos }\end{array}$ & $6(9,8)$ & $30(49,2)$ & $23(39,0)$ \\
Parâmetros laboratoriais & $19(31,1)$ & $23(37,8)$ & $17(28,8)$ \\
Sepse & & & \\
Parâmetros clínicos & $14(23,0)$ & $26(42,6)$ & $19(31,1)$ \\
Parâmetros laboratoriais & $16(26,2)$ & $28(47,5)$ & $15(24,6)$ \\
\hline & & Fonte: Dados primários da pesquisa 2015-2016
\end{tabular}

Tabela 2. Resultados do preenchimento das definições de sepse grave e choque séptico de acordo com o Consenso de Sepse pelos médicos residentes $(n=59)$

\begin{tabular}{lcc}
\hline Definições & Correto & Resultados \\
& $\mathrm{f}(\%)$ & $\begin{array}{c}\text { Incorreto } \\
\mathrm{f}(\%)\end{array}$ \\
\hline Sepse grave & $26(44,1)$ & $33(55,9)$ \\
Choque séptico & $16(27,7)$ & $43(72,4)$ \\
\hline
\end{tabular}

Tabela 3. Pontuações sobre SIRS alcançadas pelos médicos residentes do Hospital Universitário Lauro Wanderley no preenchimento do questionário sobre conhecimento do Consenso de Sepse por especialidade $\underline{(\mathrm{n}=59)}$

\begin{tabular}{|c|c|c|c|}
\hline \multirow{2}{*}{$\begin{array}{c}\text { Especialidades } \\
\left(\mathrm{n}^{0} \text { residentes }\right)\end{array}$} & \multicolumn{3}{|c|}{ Frequências de Acertos } \\
\hline & $\begin{array}{c}\text { Menos acertos } \\
4-6 \text { pontos } \\
\text { f }(\%)\end{array}$ & $\begin{array}{c}\text { Acertos intermediários } \\
7-9 \text { pontos } \\
\mathrm{f}(\%)\end{array}$ & $\begin{array}{c}\text { Mais acertos } \\
10-12 \text { pontos } \\
\text { f }(\%)\end{array}$ \\
\hline Clínica Médica (18) & $1(5)$ & $2(11)$ & $15(83,0)$ \\
\hline Cirurgia Geral (8) & - & $3(37,5)$ & $5(62,5)$ \\
\hline Pediatria (8) & - & $1(12,5)$ & $7(87,5)$ \\
\hline Infectologia (3) & - & $1(33,3)$ & $2(66,6)$ \\
\hline Ginecologia (6) & $1(16,6)$ & $3(50,0)$ & $2(33,3)$ \\
\hline Endoscopia (1) & - & $1(100)$ & - \\
\hline Anestesiologia (3) & $1(33,3)$ & - & $2(66,6)$ \\
\hline Oftalmologia (5) & $2(40,0)$ & $3(60,0)$ & - \\
\hline Reumatologia (1) & - & - & $1(100)$ \\
\hline Endocrinologia (2) & - & $1(50,0)$ & $1(50,0)$ \\
\hline Dermatologia (4) & - & $1(25,0)$ & $3(75,0)$ \\
\hline
\end{tabular}

Fonte: Dados primários da pesquisa 2015-2016

uso de critérios estritos para definir disfunção orgânica em programas de melhoria de qualidade em nosso país"14. Assim, no momento, ainda há controvérsias no Brasil e na América Latina quanto ao novo consenso Sepsis, considerando-se que aspectos das novas definições não deveriam ser inteiramente aplicáveis pelo risco de uma redução da sensibilidade e de retardo no reconhecimento da sepse em países com recursos limitados ${ }^{1}$. Contudo, nesse estudo, que foi realizado seis meses antes da publicação das novas definições, aborda-se o conhecimento do consenso internacional de sepse, adotado pela AMIB e pela ILAS, enfocandose na presente discussão, o conhecimento dos médicos residentes do HULW acerca daquele consenso.

Os resultados observados sugerem que o fato de os médicos residentes afirmarem ter conhecimento a respeito daquele consenso corrobora estudo anterior realizado em Porto Rico em que a mesma proporção de respondentes de uma amostra de médicos da área de medicina interna e cirurgia geral também relataram saber de sua existência ${ }^{15}$. Ainda que constituíssem uma amostra de profissionais que não estavam mais em formação pós-graduada, mas sim médicos atuantes em clínica e cirurgia gerais, o percentual de respostas afirmativas sobre o conhecimento foi praticamente o mesmo (90\%).

No presente estudo, o contato inicial da maioria dos residentes entrevistados com o consenso se deu durante a própria graduação, o que sugere uma adequação dos conteúdos curriculares em relação ao tema dos critérios diagnósticos de sepse então vigentes. Além disso, uma parcela elevada dos participantes afirmou aplicar o 
consenso de sepse em suas atividades práticas como médico residente do HULW. Dentre os entrevistados, a maioria atuava nas áreas de Clínica Médica e Cirurgia Geral, os quais mais frequentemente se defrontam com casos de sepse e suspeitas de SIRS em seus pacientes, diferentemente das outras subespecialidades que pouco utilizam os critérios por não os aplicar habitualmente na sua prática ${ }^{15}$.

Estimaram-se em mais de cinco casos de SIRS acompanhados pela maior parte dos médicos, sendo a maioria vivenciada na unidade de terapia intensiva (UTI) do hospital universitário. Tais dados podem ser relacionados à dificuldade de a equipe assistente do hospital para reconhecer precocemente a sepse, e mesmo a SIRS, uma vez que sua apresentação é constituída por alterações sutis e inespecíficas dos sinais vitais, diferentemente de outros quadros agudos graves como o infarto agudo do miocárdio ou do acidente vascular cerebral. Ou seja, as manifestações inespecíficas da SIRS/sepse não são marcadas por um ictus, o que dificulta sua percepção precoce e o encontro dos casos mais frequentemente em ambientes de cuidado intensivo ${ }^{8}$.

Por outro lado, esses locais onde foram observados mais frequentemente os casos de sepse também se relacionam à maior complexidade dos pacientes atendidos no nível terciário de atenção à saúde. Além disso, o risco de sepse cresce significativamente com o maior número de critérios de SIRS presentes e que, no ambiente de UTI, onde há maior vigilância clínica e monitoramento constante, favorece a detecção dos sinais de alarme, o que não é feito de forma habitual nas enfermarias ${ }^{15}$.

Observou-se nesta pesquisa que o tipo de SIRS predominantemente acompanhado pelos residentes foi a sepse, embora as pontuações obtidas através da aplicação do questionário de avaliação dos conhecimentos do consenso tenham revelado maior escore nas respostas ao item referente à SIRS que no de sepse, ainda que sem diferença estatística significativa. Há obstáculos para a detecção ainda na fase denominada de SIRS, um estágio mais precoce e de sinais clínicos inespecíficos e sem um foco de infecção evidente, havendo sinais apenas relativos à alteração nos sinais vitais e no leucograma. Esta inespecificidade, que foi uma das razões que levaram a que fosse excluída a definição de SIRS no último consenso internacional de 2016 (Sepsis 3), não representou a dificuldade preponderante de resposta apresentada pelos residentes entrevistados, embora tenha sido mais experimentada na prática a vivência clínica de detecção de sepse. No consenso de 1991, emanado da Conferência Internacional de Consenso de Sepse, SIRS é uma categoria diagnóstica que faz parte da sepse, mas ainda sem a evidência de um foco infeccioso.

Embora seja previsível um melhor conhecimento sobre critérios de SIRS/sepse com o aumento dos anos de treinamento clínico, o estudo de avaliação de conhecimento sobre o consenso de sepse referido anteriormente $^{15}$ demonstrou que não houve diferença nos acertos entre os residentes do primeiro, segundo e terceiro anos da pós-graduação, o que também é corroborado neste estudo, em que o conhecimento de SIRS e sepse apresentaram uma pontuação levemente maior nas respostas dos residentes do segundo ano em relação aos do primeiro, mas estas diferenças não foram suficientemente amplas para se inferir que residentes mais adiantados revelaram maior conhecimento que os do primeiro ano.

Por outro lado, ainda que a maioria dos residentes entrevistados tenha referido conhecimento sobre o consenso de sepse, os percentuais de acertos na avaliação de seus conhecimentos não podem ser considerados satisfatórios, levando-se em conta os percentuais de acertos na aplicação do questionário. O maior número de acertos no escore de SIRS ocorreu entre os residentes de Clínica Médica, enquanto residentes de outras especialidades, como oftalmologia e dermatologia, obtiveram porcentagens inferiores de acerto. Para o escore de sepse, os resultados foram semelhantes, o que se deve ao fato de que são os mesmos critérios, porém com a diferença de que na sepse há evidência detectável de infecção.

No quesito sepse grave, que constitui um conceito considerado atualmente como redundante, por ser a sepse tida sempre como grave, os residentes obtiveram mais acertos que em relação ao choque séptico, que é definido no consenso então em vigor como o quadro de insuficiência circulatória que não responde às medidas de reposição volêmica. Sepse grave é uma terminologia que foi abolida no último consenso internaciona ${ }^{11}$, e atualmente é denominada apenas como sepse, que se refere a uma infecção suspeita ou confirmada associada a disfunção orgânica, de forma independente da presença de sinais de SRIS, segundo o Instituto Latino Americano de Sepse ${ }^{10}$, revisado em 2017.

No entanto, na amostra como um todo, a elevada proporção de respostas incorretas referentes à definição de choque séptico, é inquietante, considerando-se as altas taxas de mortalidade dos casos enquadrados nesta categoria mais avançada da insuficiência circulatória. No entendimento atualmente ainda aceito no Brasi ${ }^{10}$, choque séptico denomina a sepse que evoluiu com hipotensão não corrigida com reposição volêmica (PAM $\leq 65 \mathrm{mmHg}$ ).

Resultados semelhantes ao dessa investigação científica foram encontrados em estudo indiano ${ }^{16}$, que avaliou o conhecimento de 136 médicos residentes de clínicas médica e cirúrgica de um hospital na Índia sobre o consenso de sepse, demonstrando-se que em relação às definições de sepse grave e choque séptico, apenas $32,4 \%$ dos residentes conseguiram acertar os conceitos operacionais da síndrome. Também de forma semelhante 
aos resultados do presente estudo, os referidos autores verificaram que os residentes de cirurgia tiveram uma taxa menor de respostas corretas do que os residentes de clínica médica.

Em estudo para avaliar o conhecimento dos profissionais médicos que trabalham em unidades de emergência e de terapia intensiva sobre as definições da SIRS, sepse, sepse grave e choque séptico dos hospitais de grande porte da cidade de Florianópolis$\mathrm{SC}^{11}$, observou-se um conhecimento considerado adequado, com $54,8 \%$ de acertos, um percentual superior ao encontrado no presente estudo. Contudo, a amostra avaliada no referido estudo foi composta por profissionais já pós-graduados atuantes em medicina intensiva e emergência médica, e não por médicos ainda residentes. Mas de forma similar ao que foi observado na nossa amostra, SIRS e sepse foram os quadros referidos como mais conhecidos, enquanto sepse grave e choque séptico apresentaram menor porcentagem de acertos.

Por outro lado, em estudo em que se avaliou o conhecimento de profissionais de Enfermagem e de Medicina na identificação das ocorrências de sepse em uma unidade de urgência e emergência de um hospital público na cidade de Curitiba-PR ${ }^{12}$, verificou-se um conhecimento inadequado pelos profissionais, tanto no reconhecimento de sinais e sintomas relacionados a SIRS e a disfunção orgânica, como também na identificação dos quadros clínicos, pois apenas $17,4 \%$ responderam corretamente. Assim, similarmente aos dados deste estudo, neste último trabalho referido também houve um conhecimento inadequado do consenso de sepse pelos profissionais. Ainda em estudo com profissionais médicos plantonistas na UTI e no Pronto Socorro de um hospital de Goiás, constatou-se que $66,6 \%$ utilizavam o preconizado pelo ILAS enquanto $22,2 \%$ não tinham conhecimento da existência de um consenso ou protocolo a ser seguido por todos no local de trabalho ${ }^{17}$.

Embora se possa considerar que o mais importante é que os médicos saibam quais os dados clínicos e laboratoriais que caracterizem SIRS e sepse, e não necessariamente conhecer as definições das mesmas, é necessária capacitação para o reconhecimento dos sinais de sepse. A sepse, um problema de saúde pública, se constitui doença aguda grave e de alta mortalidade, que exige das instâncias relacionadas à educação médica esforços para educação permanente nesse sentido, assim como à promoção de campanhas de conscientização junto aos profissionais de saúde e melhoria da infraestrutura para o atendimento $0^{7,12,18}$.

A estratificação de critérios para reconhecimento da sepse na prática clínica é considerada uma diretriz relevante a ser seguida visando à identificação de necessidades de monitorização, suporte orgânico e controle do foco ${ }^{19}$. A capacidade para identificar um paciente com sepse é vital para que este apresente o melhor desfecho possível e, nesse sentido, definições e critérios são muito relevantes.

\section{Considerações finais}

Conclui-se que a grande maioria dos residentes do HULW refere ter conhecimento acerca do Consenso de Sepse, adquirido sobretudo na graduação, e que o colocam em prática em suas atividades cotidianas, em um processo de aperfeiçoamento profissional que constitui objetivo principal da residência médica. Contudo, a avaliação do conhecimento sobre SIRS/ sepse revela que os percentuais de respostas corretas em parâmetros clínicos e laboratoriais foram insatisfatórios, observando-se mais respostas incorretas em relação aos parâmetros clínicos, assim como aquelas referentes ao conceito de choque séptico. Houve mais acertos entre os residentes da clínica médica e pediatria, assim como entre os residentes do segundo ano.

Reitera-se a existência de dificuldade em se diagnosticar precocemente a sepse e que mais instruções sobre o gerenciamento da sepse são necessárias como parte do processo de educação continuada. Os médicos residentes necessitam ser treinados para o reconhecimento dos sinais de sepse, o que inclui o conhecimento das definições de consenso, sejam quais forem os parâmetros vigentes.

\section{Referências}

1. Singer M, Deutschman CS, Seymour CW, Shankar-Hari M, Annane D, Bauer $\mathrm{M}$ et al. The Third International Consensus Definitions for Sepsis and Septic Shock (Sepsis-3). JAMA. 2016; 315(8):801-10.

2. Arefian H, Heublein S, Scherag A, Brunkhorst FM, Younis MZ, Moerer $\mathrm{O}$ et al Hospital-related cost of sepsis: A systematic review. J Infect. 2017;74(2):107-117.

3. Barreto MFC, Dellaroza MSG, Kerbauy G, Grion CMC. Sepsis in a university hospital: a prospective study for the cost analysis of patients' hospitalization. RevEscEnferm USP • 2016; 50(2):299-305

4. Santos AM, Souza GRB, Oliveira AML. Sepse em adultos na unidade de terapia intensiva: características clínicas. Arq Med Hosp Fac Cienc Med Santa Casa São Paulo. 2016; 61:3-7.

5. Silva BL, Ribeiro FF, Andrade SSC, Fonsêca LCT. Morbimortalidade Hospitalar por Sepse no Sistema Único de Saúde. Revenferm UFPE online. 2013; 7(1):23-9

6. ILAS. Instituto Latino Americano de Sepse. Sepse: um problema de saúde pública / Instituto Latino-Americano de Sepse. Brasília: CFM, 2015. http:/ www.ilas.org.br/assets/arquivos/upload/Livro-ILAS(Sepse-CFM-ILAS). pdf. Acesso em 18 agosto de 2018

7. Vincent JL, Mira JP, Antonelli M. Sepsis: older and newer concepts. Lancet Respir Med. 2016;4(3):237-40.

8. Balk RA. Systemic inflammatory response syndrome (SIRS): where did it come from and is it still relevant today? Virulence 2014;5(1):20-6

9. Horeczko T, Green JP, Panacek EA. Epidemiology of the Systemic Inflammatory Response Syndrome (SIRS) in the emergency department. West J Emerg Med. 2014;15(3):329-36.

10. ILAS. Instituto Latino Americano de Sepse. Implementação de Protocolo Gerenciado de Sepse Protocolo Clínico. 2017. http://www.ilas.org.br/assets/ arquivos/ferramentas/protocolo-de-tratamento.pdf.2017. Acesso em: 18 ago. 
2018.

11. Pizzolatti ML, Moritz RD, Andrade J. Avaliação do Conhecimento dos Profissionais da Área de Medicina de Urgência sobre os Critérios de Definição de SIRS, Sepse, Sepse Grave e Choque Séptico. Rev Bras Ter Intensiva 2004; 16(4):210-4.

12. Melech CS, Paganini MC. Avaliação do Conhecimento de Médicos e Equipe de Enfermagem nas Ocorrências de Sepse. Rev. Med. UFPR 2016; $3(3): 127-132$.

13. Matos GFJ, Victorino JA. Critérios para o Diagnóstico de Sepse, Sepse Grave e Choque Séptico. Rev Bras Ter Intensiva 2004; 16 (2): 102-104.

14. Machado FR, Assunção MSC, Cavalcanti AB, Japiassú AM, Azevedo LCP, Oliveira MC. Chegando a um consenso: vantagens e desvantagens do Sepsis 3 considerando países de recursos limitados. Rev Bras Ter Intensiva 2016;28(4):361-365

15. Fernandez R, Boque, M, Galera A, Rodriguez W, Torres-Palacios A, Rodriguez-Vega G et al. Sepsis: A study of physicians' knowledge about the surviving sepsis campaign. Critical Care Medicine 2006; 33(12): 160.

16. Suntornlohanakul O, Khwannimit B. Comparison of Residents' Knowledge Regarding the Surviving Sepsis Campaign 2012 Guideline. Indian Journal of Critical Care Medicine 2017; 1 (2): 69-74.

17. Castro APN, Mourão DTV, Mendonça US, Silva AM. Análise da conduta dos profissionais de saúde frente à sepse. RESU - Revista Educação em Saúde 2015; 3 (2): 30-34.

18. Kaukonen KM, Bailey M, Pilcher D, Cooper DJ, Bellomo R. The systemic inflammatory response syndrome criteria and their differential association with mortality. J Crit Care. 2018;46:29-36.

19. Dias FS. Definições de sepse. Rev Bras Ter Intensiva 2017; 29 (4): 520521. 